Guideline: Draft for Consultation, June 2008. NICE (http: / / www. nice.org.uk/nicemedia/pdf/BorderlinePersonalityDisorderNICEGuidelineForConsultation.pdf).

Pearce, S. \& Haigh, R. (2008) Mini therapeutic communities - a new development in the United Kingdom. Therapeutic Communities, in press.

Scott, L. \& Attwood, G. (2008) Integrative psychotherapeutic group work: a way forward in the treatment of personality disorders. Quarterly Journal of Mental Health, in press.

Lisle M. A. Scott Psychiatrist, Oxfordshire Complex Needs Service (email: Lisle.Scott@obmh.nhs.uk); Elizabeth Kemp Counselling Psychologist, Older Adults' Service, Oxfordshire and Buckinghamshire Mental Health NHSFoundation Trust, UK

doi: 10.1192/apt.14.5.399

\section{Catatonia in present day society}

Rajagopal (2007) suggests that catatonia is a unique syndrome that requires treatment in its own right, independent of any underlying disorder.

We conducted a postal survey of psychiatrists working in south-west England and in Wales, looking at their understanding of catatonia. We also enquired about the number of cases of catatonia encountered in a 2-year period, the presence of underlying/comorbid disorders, treatment type and response to treatment.

A large number of varied signs are associated with the syndrome (Bush et al, 1996) and they may be subtle and unrecognised. No specific diagnostic criteria for catatonia have been established, although most authors define the syndrome by the presence of motor signs. There is a growing evidence base for effective treatment, and recognition of catatonia is important to prevent significant morbidity (Rosebush \& Mazurek, 1999).

In our survey, $96 \%$ of respondents understood catatonia as a syndrome in its own right and as a subtype of schizophrenia. For the majority of signs, $90 \%$ of respondents felt confident to give a definition but there was no consensus regarding which signs need to be present to diagnose catatonia. Only $28 \%$ of respondents reported seeing a case of catatonia and a range of different treatments were used.

Catatonia continues to be recognised, but the understanding of the condition differs, which could result in underdiagnosis and suboptimal treatment. The use of rating scales might ensure more that cases are identified. There is a need to raise awareness among clinicians of this often forgotten disorder.

Bush, G., Fink, M., Petrides, G., et al (1996) Catatonia. I. Rating scale and standardized examination. Acta Psychiatrica Scandinavica, 93, 129-136.

Rajagopal, S. (2007) Catatonia. Advances in Psychiatric Treatment, 13, 51-59.

Rosebush, P. I. \& Mazurek, M. F. (1999) Catatonia: re-awakening to a forgotten disorder. Movement Disorders, 14, 395-397.

Sally A. Morgan Specialist Registrar in General Adult Psychiatry, CRHTT, 18 Denmark Road, Gloucester GL1 3HZ, UK (email: sallymorgan2000@yahoo.co.uk); Dietmar Hank Visiting Honorary Consultant in Neuropsychiatry, Danny Rogers Consultant Neuropsychiatrist, The Burden Centre, Frenchay Hospital, Bristol, UK

doi: 10.1192 /apt.14.5.400 